Document downloaded from:

http://hdl.handle.net/10251/176797

This paper must be cited as:

Bessini, J.; Shepherd, P.; Monleón Cremades, S.; Lazaro, C. (2020). Design of bendingactive tied arches by using a multi-objective optimization method. Structures. 27:2319-2328. https://doi.org/10.1016/j.istruc.2020.07.045

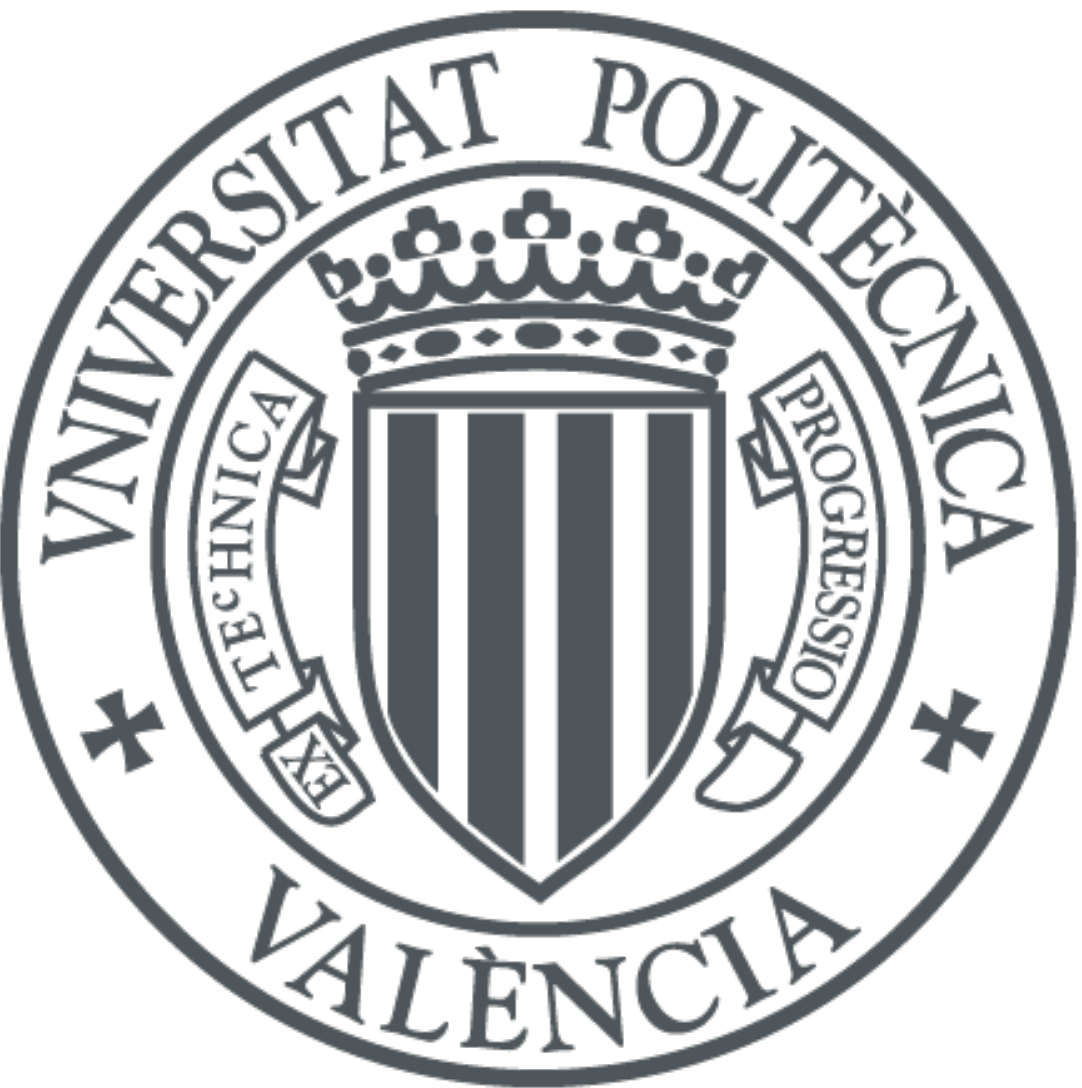

The final publication is available at

https://doi.org/10.1016/j.istruc.2020.07.045

Copyright Elsevier

Additional Information 
This paper must be cited as:

Bessini, J.; Shepherd, P.; Monleón, S.; Lázaro, C. (2020). Design of bending-active tied arches by using a multi-objective optimization method. Structures. Vol. 27, pp. 2319-2328.

doi: 10.1016/j.istruc.2020.07.045

The final publication is available at:

https://doi.org/10.1016/j.istruc.2020.07.045.

Copyright

Elsevier 
This work is licensed under a Creative Commons "Attribution-NonCommercial-NoDerivatives 4.0 International'" license.

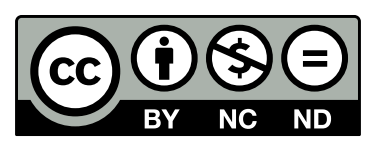




\title{
Design of bending-active tied arches by using a multi-objective optimization method
}

\author{
J. Bessini ${ }^{\mathrm{a}, *}$, P. Shepherd ${ }^{\mathrm{b}}, \mathrm{S}$. Monleón $^{\mathrm{c}}$, C. Lázaro ${ }^{\mathrm{c}}$ \\ ${ }^{a}$ TYPSA Consulting Engineers \& Architects, C/ Botiguers, 5 - $5^{\circ} 46980$ - Paterna, Spain \\ ${ }^{b}$ Department of Architecture \& Civil Engineering, University of Bath, Bath BA2 7AY, UK \\ ${ }^{c}$ Departamento de Mecánica de Medios Continuos y Teoría de Estructuras, Universitat \\ Politècnica de València, Camino de Vera s/n, 46022 Valencia, Spain
}

\begin{abstract}
The design of bending-active structures is a challenging problem, due to the high non-linearity of the activation process, the coupling between member sizing, structural shape and the deformability and buckling sensitivity inherent in the resulting lightweight configurations. Due to the large number of formfinding variables, the choice of member sizing is one of the main difficulties at the conceptual phase. In this paper, authors propose a design tool to generate efficient structural configurations for braced bending-active tied arches using multi-objective optimization strategies. Initially, a non-linear FE analysis is performed for each plausible configuration and at each generation of the optimization algorithm. In a second step, a genetic algorithm classifies the solutions and establishes new structural configurations according to best performance. Solutions are evaluated in terms of stresses in the active member and cables, and maximum deflections, as required by design codes for pedestrian bridges. Results are given in terms of non-dimensional parameters, in order to make them applicable to a wide variety of scales.
\end{abstract}

Keywords: active-bending, multi-objective optimization method, genetic algorithm, bending-active tied arch

\footnotetext{
* Corresponding author

Email address: jbessini@typsa.es (J. Bessini )
} 


\section{Introduction}

Active bending is currently attracting considerable attention as an experimental typology for lightweight structures, both in research and practice. It constitutes a structural type in which certain flexible structural members are

5 initially bent into curved shapes and then stabilized by additional cables or structural elements to form a complete structural system. Since the construction of the Mannheim Multihalle (1976) [1, a number of dome-shaped gridshells have been built; many of them as temporary or experimental structures [2, 3, 4, 5, 6, 7, 8, 9. However, the literature on their structural performance

10 and efficiency in relation to their shape and member sizing is still limited.

When designing bending-active structures, a compromise between stiffness and flexibility must be found. Curved members must be slender enough to keep activation stresses low. However, designing with very slender members may lead to structures with low stiffness. Indeed, many bending-active gridshells that need to support heavier loads, such as footbridges, the design space may be very limited, and this explains why there are very few bending-active examples.

Lienhard [10] studied the efficiency of elastica-shaped arches with different rise-to-span ratios subject to simple loading patterns. Douthe [11 analyzed the practicality of different materials for active grid-shell members in accordance with their strength and stiffness. The authors [12] analyzed the response of circular and elastica-shaped active arches subject to a point load, and quantified the relation between geometric stiffness, tangent stiffness and the rise-to-span ratio for different values of rod slenderness. More recently, the authors presented

${ }_{25}^{25}$ 13, 14 a preliminary study of the performance and practicality of bendingactive tied arches with bracing. This involves simple planar structures composed of a continuous flexible member that is activated by the action of main cables pulling at both ends of the rod, and secondary struts that deviate the main cable and act at certain points along the rod (Fig. 1).

Sakai et al. [15] presented a methodology to design gridshells using elastica- 
shaped arches. This contribution is worth highlighting because it is novel work in the implementation of optimization techniques to achieve efficient systems for bending-active structures. The optimization approach is aimed at obtaining structural configurations with small interaction forces between beam elements 35 at joints.

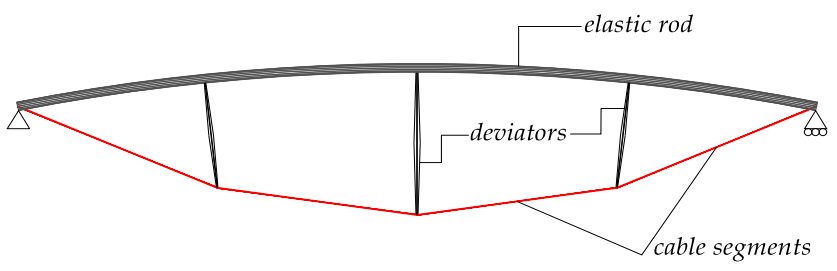

Figure 1: Bending-active tied arch

The interest of simple arches with bracing lies in their capacity as resistant schemes to design lightweight pedestrian bridges [9] or roofing applications [16]. Using this experimental structural scheme, the authors have designed and built a $5 \mathrm{~m}$ long prototype of an experimental lightweight footbridge (Fig. 2). The 40 system is composed of a pair of planar bending-active tied arches that are independently activated and connected by hinged links at the level of the main cable and horizontal struts at the level of the rods [17.

The results obtained for the performance of bending-active tied arches systems 45 for pedestrian bridge applications show that the region of the design space where solutions comply with the design constraints is fairly limited, due primarily to the magnitude of the design loads and the tight limitations on stress and deflection posed by codes. Solutions are dominated by instability in the active members, minimum stresses in cables after activation and maximum allowable deflections for the serviceability limit state.

In previous work [14, the authors carried out a series of simulations using specific sizes of members and material properties, for a certain length ratio between deviators. However, the results obtained from this study are not generally 


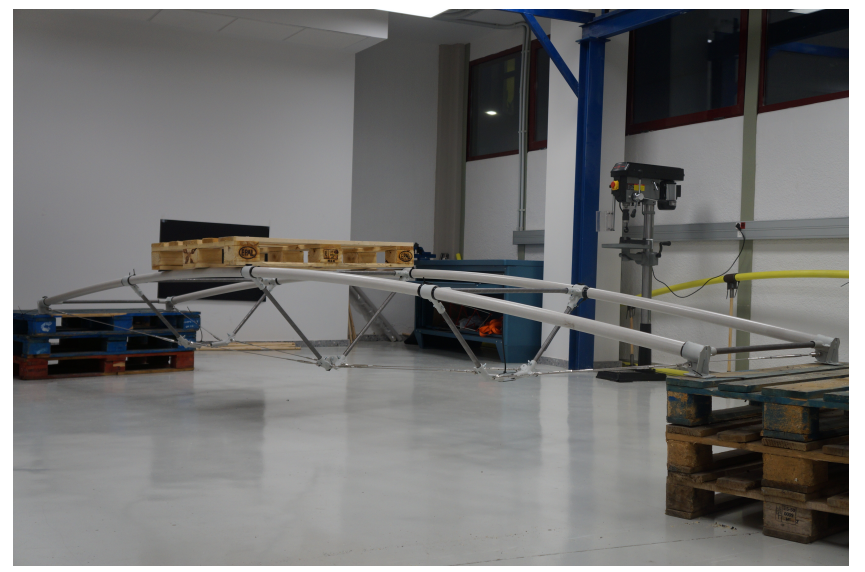

Figure 2: Experimental lightweight footbridge based on the active bending principle

applicable to bending-active tied arches with different geometries and member

${ }_{55}$ proportioning, since it would be necessary to replicate the numerical experiments for every potential structural configuration. Due to the large number of form-finding parameters, and the restrictive limitations posed by codes, the determination of the best structural configuration is a challenging process.

In this paper, the authors propose a design tool to obtain efficient structural configurations for bending-active tied arches using multi-objective optimization strategies. In a first step, plausible random configurations are generated by the combination of different form-finding parameters. In a second step, such configurations are simulated using a non-linear analysis software for the tensioning process, and subject to the serviceability and ultimate limit state in accordance with the limitations posed by the Eurocode for footbridges. In a third step, a genetic algorithm classifies the solutions and establishes new structural configuration according to best performance. Finally, results are given in terms of non-dimensional parameters, which make them applicable to a wide variety of scales and cross-sectional sizes.

70 The outline of the paper is as follows: In section 2 the problem is introduced and the considered variables in the multi-objective optimization method are detailed. In section 3 the genetic algorithm is described and a sensitivity study is 
carried out to calibrate the method. The results obtained after the optimization process are shown in section 4 . In section 5 the solution is analyzed from a

75 structural point of view. Finally, conclusions are outlined in section 6 .

\section{Problem description}

This study focuses on symmetric bending-active tied arches with three equally spaced deviators, which remain perpendicular to the rod no matter whether they work under compression or tension. The following common parameters have been considered: the upper rod is a $4 \mathrm{~m}$ long continuous member with circular hollow cross-section; cables are modelled using solid cross-sections -which are equivalent to the cross-sectional area of a wire rope- and are not continuous; therefore, cable forces can be different in each cable segment; deviators are modelled as rigid bodies. The design of deviators is beyond the scope of the present study. They should be defined in a second stage once the shape of the structure and the equilibrium forces are obtained. The material properties of the rod and cables are shown in Table 1 , where $E$ is the elastic modulus and $f_{u}$ is the ultimate strength of the selected material.

\begin{tabular}{rccc}
\hline Element & Material & $E(\mathrm{MPa})$ & $f_{u}(\mathrm{MPa})$ \\
\hline Rods & GFRP & 30000 & 400 \\
Cables & Steel & 110000 & 1570 \\
\hline
\end{tabular}

Table 1: Material properties

There are five main form-finding parameters in this problem: the size and cross-sectional shape of the rod, the diameter of the cables and the deviator lengths at midspan and at quarters. To populate the data set of the multiobjective optimization problem, some of these parameters are introduced as non-dimensional variables. In the following, the input variables are detailed:

- The diameter and thickness of the circular hollow cross-section of the rod are given by the selected outer $D_{e}$ and inner $D_{i}$ diameters. The outer 
diameter can vary from $s / 20$ to $s / 8$, where $s$ is the length of the rod segment between deviators; the inner radius is given by the ratio $D_{i} / D_{e}$, which can vary from 0.2 to 0.8 . These limitations allow to get light activemembers without risk of local buckling or crushing under the effect of external loads.

- The length of the deviator at midspan $h_{m}$ can range from $L / 15$ to $L / 5$, where $L$ is the developed length of the rod. The length of the deviators at quarter-points $h_{q}$ is defined by means of the ratio $h_{q} / h_{m}$. These geometrical constraints are selected from an aesthetic point of view.

- The diameter of cables $d$ is given in terms of cable-rod slenderness ratio $\bar{\lambda}_{c} / \bar{\lambda}$. The definition of slenderness is inherited from the expression of mechanical slenderness stated in the Eurocode 3 (see equation (6.5) in [18]). The slenderness of the rod is defined as $\bar{\lambda}=\frac{s}{\pi} \sqrt{\frac{A}{I}} \sqrt{\frac{f_{u}}{E}}$, where $A$ is the cross-sectional area, $I$ is the moment of inertia, $f_{u}$ is the ultimate strength of the selected material and $E$ is its elastic modulus. The cable slenderness parameter is characterized as $\overline{\lambda_{c}}=\frac{s}{d} \sqrt{\frac{f_{u_{s}}}{E_{s}}}$, where $f_{u_{s}}$ and $E_{s}$ are the ultimate strength and elastic modulus of the steel respectively. Using the cable-rod slenderness ratio $\bar{\lambda}_{c} / \bar{\lambda}$ as a parameter to define the size of the cable makes it possible to avoid configurations with oversized or undersized diameters with respect to the size of the rod, which is undesirable for the global behavior of the structure [14]. For example, Fig. 3 shows two configurations with the same rod slenderness value $\bar{\lambda}=1.5$ but with different cable-rod slenderness ratios $\overline{\lambda_{c}} / \bar{\lambda}$, which correpond with the lower and upper bounds 5 and 15 respectively.

Table 2 summarizes the bounds chosen for the form-finding parameters (Fig. (4).

The design of the bending-active tied arch must also meet the functional requirements for footbridges posed by codes. Therefore, the analysis is restricted to arches with a rise-to-span ratio $f / a$ close to $6 \%$ (Fig. 4). This value corre125 sponds with the maximum allowable gradient of the deck. 


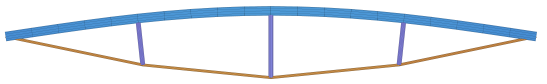

(a)

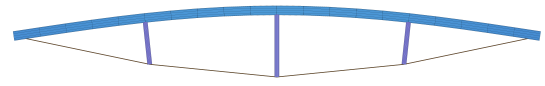

(b)

Figure 3: Configurations with the same rod slenderness $\bar{\lambda}=1.5$ and different cable-rod slenderness ratios: a) $\overline{\lambda_{c}} / \bar{\lambda}=5$ and b) $\overline{\lambda_{c}} / \bar{\lambda}=15$

\begin{tabular}{rcc}
\hline Variable & Lower bound & Upper bound \\
\hline$h_{m}$ & $L / 15$ & $L / 5$ \\
$h_{q} / h_{m}$ & 0.3 & 1 \\
$D_{e}$ & $s / 20$ & $s / 8$ \\
$D_{i} / D_{e}$ & 0.2 & 0.8 \\
$\bar{\lambda}_{c} / \bar{\lambda}$ & 5 & 15 \\
\hline
\end{tabular}

Table 2: Upper and lower bounds for the input variables in the multi-objective optimization method

The design and analysis of bending-active structures must be evaluated at two different stages: the activation phase and the serviceability limit state. The first corresponds to the tensioning process, where elastic members such as rods or beams, which are initially straight and unstressed (Fig. 5), are bent by introducing a force $T^{0}$ in the outer cable (Fig. 4). Perpendicularity between rod and deviators is achieved by selecting the corresponding force $T^{1}[14$.

During this phase, there is a strong interaction among member sizes, tensioning forces and material properties, involving large displacements and rotations of

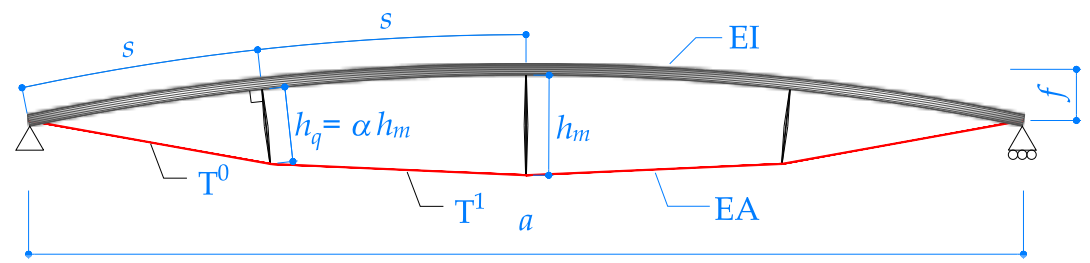

Figure 4: Definition of the input parameters 


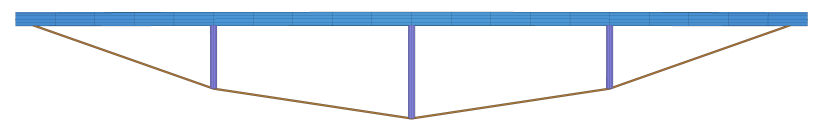

Figure 5: Initial flat state before the tensioning process

the rod cross-sections. In previous work [14, the authors proved that activation forces can be obtained by the relation

$$
T^{0}=\left(\frac{\pi}{l_{c}}\right)^{2} E I
$$

where $l_{c}$ acts as a shape parameter and the bending stiffness of the rod $E I$ determines the magnitude of the activation force $T^{0}$. Therefore, the shape of the arch can be adjusted independently of the member sizes by keeping constant the relation

$$
\left(\frac{\pi}{l_{c}}\right)^{2}
$$

Non-linear analysis is required in order to simulate the tensioning process, since the equilibrium configuration cannot be defined 'a priori'. Simulations have been carried out using the non-linear Finite Element (FE) software SOFiSTiK.

135 With the aim of obtaining self-stressed configurations with a particular riseto-span ratio $f / a$, each flat configuration is simulated for four values of activation forces given by the bending stiffness of the rod $E I$ and four shape parameters $l_{c}$, which remain constant for all the experiments (Fig. 6). Secondly, the closest solution to the desired non-dimensional shape ratio $f / a$ is selected. After activation, stresses in the rod are evaluated at midspan using the axial force and bending moment. Stresses in the cables are also quantified.

The second consideration in the design of bending-active structures is the serviceability limit state. To achieve this a distributed load corresponding to $40 \%$ of the $5 \mathrm{kN} / \mathrm{m}^{2}$ service load is applied according to the loading model for

${ }_{145}$ footbridges posed by the Eurocode [19]. Deflections are calculated at midspan $\left(D_{m}\right)$ and at quarters $\left(D_{q}\right)$ using two loading patterns. 


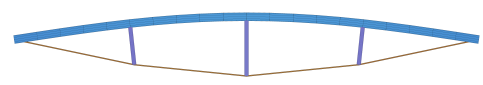

(a) $\left(\frac{\pi}{l_{c}}\right)^{2}=\frac{1}{4}$

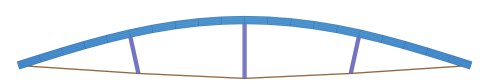

(c) $\left(\frac{\pi}{l_{c}}\right)^{2}=\frac{1}{2}$

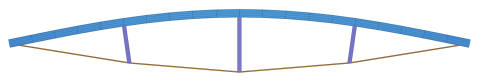

(b) $\left(\frac{\pi}{l_{c}}\right)^{2}=\frac{1}{3}$

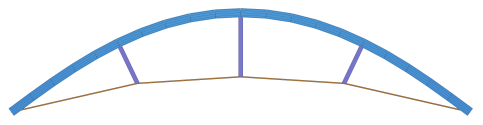

(d) $\left(\frac{\pi}{l_{c}}\right)^{2}=1$

Figure 6: Self-stressed configurations for different activation forces $T^{0}$ given by the shape parameter $\left(\frac{\pi}{l_{c}}\right)^{2}$ and the mechanical properties of the rod $E I$

The first is a symmetric uniform load on a width chosen to be $10 \%$ of the developed length of the rod (Fig. 7). The second is a non-symmetric uniform load on half-span with the same width (Fig. 8).

For the evaluation of the ultimate limit state, the characteristic load value $5 \mathrm{kN} / \mathrm{m}^{2}$ is multiplied by the partial factor for actions $\gamma=1.35[19$.

Normal forces and bending moments are assessed in the rod at midspan considering the symmetric loading pattern and performing a FE non-linear analysis.

Due to the lightness of this kind of structures, the effects caused by the self-weight can be neglected in the simulations. This simplifies the analysis and allows to be isolated the effect of external loads.

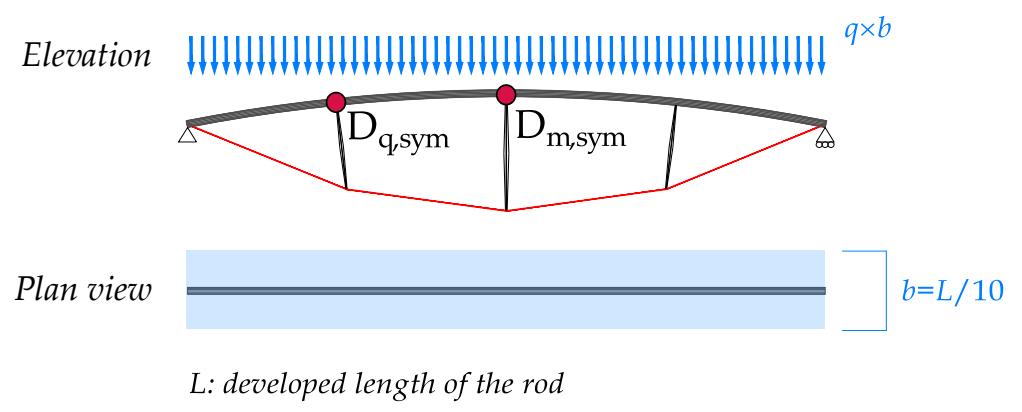

Figure 7: Symmetric loading pattern 


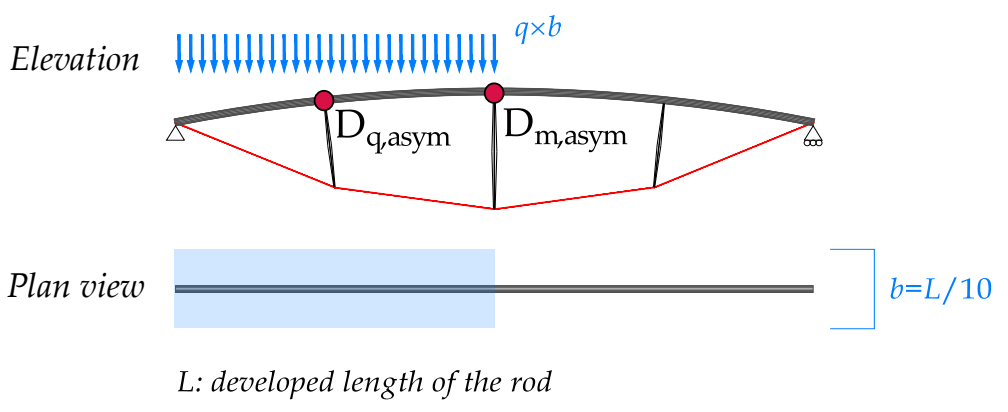

Figure 8: Non-symmetric loading pattern

\section{The multi-objective optimization problem}

The computational framework presented in this paper combines two techniques to obtain efficient structural configurations: a non-linear FE analysis and a genetic algorithm. The process starts with the definition of the set of feasible solutions (population). Individuals are randomly initialized and composed of five genes that correspond to the form-finding parameters described in Table 2 . Secondly, a non-linear FE analysis is performed for each individual and in each generation, in order to evaluate the structural response of the tied arch. Finally, the genetic algorithm carries out fitness-based selection and recombination to produce the next generation of suitable structural configurations (Fig. 9).

\subsection{Fitness}

In the design of bending-active structures, the selection of member proportions determines the behavior of the whole structure. Oversized cable crosssections can lead to insufficient stress at the activation stage, and undersized cross-sections may result in an excessively flexible system. The active member is required to be slender enough to keep stresses low after activation. Moreover, oversized rod cross-sections lead to heavy solutions that are not interesting from an aesthetic point of view. To satisfy these requirements, four fitness objectives 175 (cost functions) are considered in the proposed multi-objective minimization 


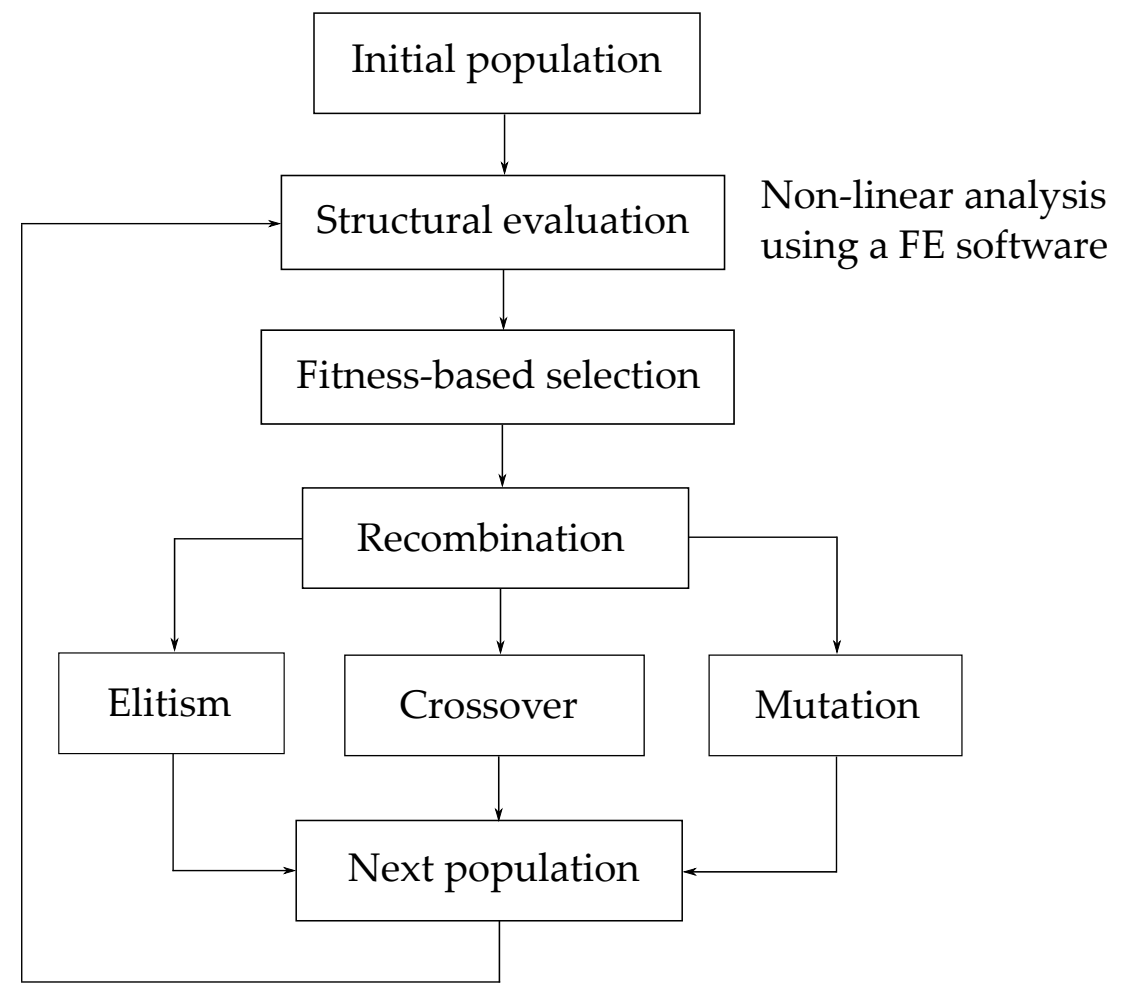

Figure 9: Flowchart of the design method 
problem to evaluate each structural configuration. Fitness function depends on: a) the utilization ratio of the rod for the ultimate limit state $R_{U L S}$; b) the utilization ratio of cables after activation and for the serviceability limit state $C_{F F-S L S}$, where the resulting cable cross-section should be capable of reaching 180 at least $10 \%$ of the maximum allowable stress after activation, and at most $70 \%$ under service loads; c) the maximum deflection at midspan $D_{m}$ and at quarters $D_{q}$ for serviceability limit state $D_{S L S}$, with the ratio $L / 1200$ as the target according to codes for footbridge applications; and d) the weight of the rod and cables $W$ to guide the process towards structural configurations that are as light as possible.

The utilization ratio of the rod has been calculated according to EN 1993-1-1 (see Eurocode 3, Eq. 62.2 [18]). The expression reads as follows:

$$
\frac{N}{N_{u}}+\frac{M}{M_{u}}
$$

where $N$ and $M$ are the axial force and bending moment respectively, produced either by the bending of an initial straight rod during the tensioning process or the application of external design loads; $N_{u}$ and $M_{u}$ are the design values of the ultimate axial forces and bending moments respectively, without considering buckling reduction factors. For cables, the expression is simplified due to the absence of bending moments:

$$
\frac{N}{N_{u}}
$$

The fitness score of each individual $(i)$ and at each generation $(t)$ is obtained as the weighted summation of the fitness functions (eq. 5). The weight for each fitness function has been selected according to the relative importance of the variables, defined by the authors (Fig. 10). The part of the fitness score related to serviceability limit state $F_{c}\left(D_{S L S}\right)$, has been obtained as the equally weighted summation of the fitness function $F_{c}\left(D_{l, p}\right)$ for each non-dimensional deflection $D_{l, p}$ considering the different loading patterns (symmetric and non-symmetric) 
and positions (at midspan and at quarters) (eq 6 ).

$$
\begin{aligned}
\text { Fitness }_{i}^{t}= & 0.4 F_{a}\left(R_{U L S}\right)+0.1 F_{b}\left(C_{F F-S L S}\right)+ \\
+ & 0.25 F_{c}\left(D_{S L S}\right)+0.25 F_{d}(W) \\
F_{c}\left(D_{S L S}\right) & =\frac{1}{4} F_{c_{1}}\left(D_{q, s y m}\right)+\frac{1}{4} F_{c_{2}}\left(D_{m, \text { sym }}\right)+ \\
& +\frac{1}{4} F_{c_{3}}\left(D_{q, \text { asym }}\right)+\frac{1}{4} F_{c_{4}}\left(D_{m, \text { asym }}\right)
\end{aligned}
$$
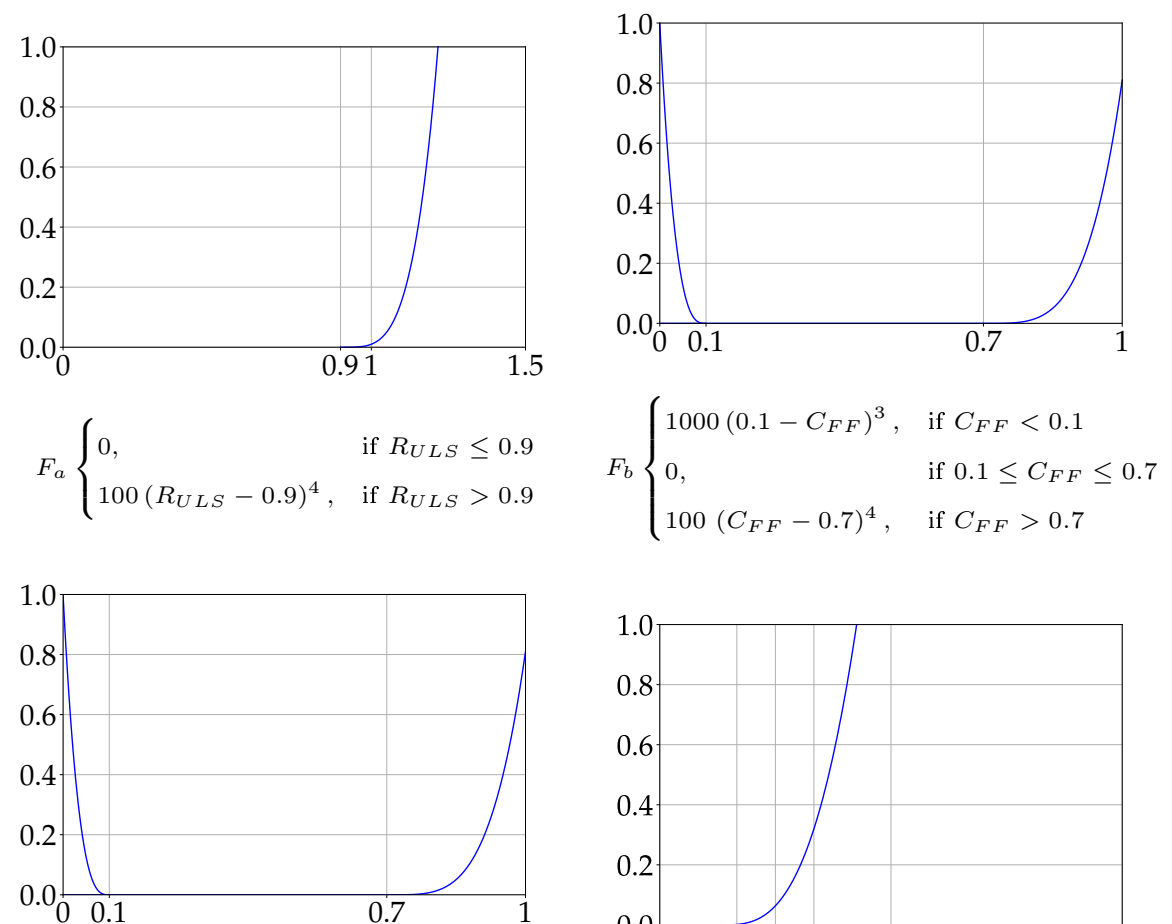

$F_{c} \begin{cases}0, & \text { if } D_{l . p} \leq \frac{L}{1200} \\ 5 \cdot 10^{6}\left(D_{l, p}-\frac{L}{1200}\right)^{3}, & \text { if } D_{l, p}>\frac{L}{1200}\end{cases}$

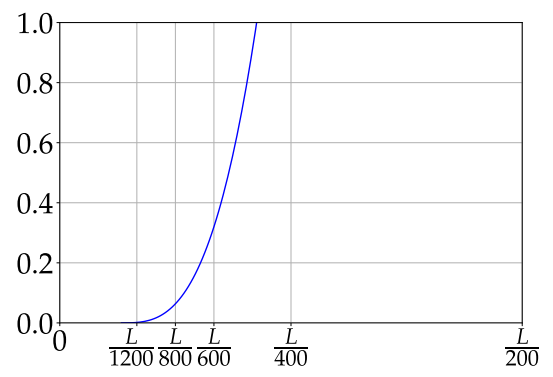

$F_{d}=1.25 W$ with $W$ in $\mathrm{kN} / \mathrm{m}$

Figure 10: Fitness functions considered in the multi-objective optimization problem

\subsection{Selection}

195 technique [20]. This selection strategy allocates each individual a probability of 
being selected, proportional to its relative fitness score, which is computed by dividing the fitness of each individual by the sum of all fitness values, normalizing to 1 . Since the optimization method is a minimization problem, we cannot apply this technique directly. Instead we use 1 - fitness, so that individuals with a lower fitness score will be more likely to be chosen as a parent. Using this technique, the best individuals can be selected multiple times for breeding.

\subsection{Recombination and evolution}

Among existing crossover techniques, the one-point crossover has been implemented in this research. Every pair of parents are each cut at a random position (crossover point) and the genes on one side of the crossover point are swapped to generate two new individuals [21]. After recombination, each individual has a $40 \%$ probability of mutation (see section 3.4). In this case, one of its genes, randomly selected, adopts a new value within the initial predefined bounds. This technique introduces diversity into the population, which prevents the algorithm from becoming trapped in a local minimum [22]. Finally, the successor population is generated using replacement with elitism, where the best individual from the current population is carried over unaltered to the next generation.

The genetic algorithm iterates until it reaches a configuration whose fitness score remains unimproved for at least 100 consecutive iterations.

\subsection{Sensitivity study}

To improve the genetic algorithm performance, a sensitivity study has been carried out to determine an efficient set of genetic algorithm parameters. The set of experiments obtain the optimum population size and probability of mutation. It has been considered that all fitness functions are equally weighted and a maximum number of iterations of 300 . In Table 3 , it can be observed that setting a large population does not lead to a better solution. On the other hand, with small populations, a probability of mutation close to $40 \%$ offers the best outcome. In addition, setting small populations is advantageous from the point of view of reducing the computational cost. 


\begin{tabular}{cccc}
\hline Experiment & Population & Mutation [\%] & Fitness $_{i}^{300}$ \\
\hline E1 & 25 & 0 & 0.0200 \\
E2 & 25 & 30 & 0.0155 \\
E3 & 25 & 40 & 0.0151 \\
E4 & 25 & 50 & 0.0156 \\
& & & \\
E5 & 50 & 40 & 0.0156 \\
& & & \\
E6 & 100 & 30 & 0.0154 \\
E7 & 100 & 40 & 0.0155 \\
\hline
\end{tabular}

Table 3: Experiments to select the population size and probability of mutation

\section{Results}

Results are given in terms of non-dimensional parameters and shown graphically to better understand the evolution of the form-finding variables through the multi-objective optimization process. Figure 11 shows the range of genome values for the initial population considered in the problem, which has been randomly defined using a Sobol sequence [23]. This method distributes the points evenly and uniformly. Figure 11 a) depicts the length of the central deviator and the length ratio between deviators; Figure $11 \mathrm{~b}$ ) provides information about the cross-section of the rod; Figure 11 c) establishes the member ratio between rod and cables. In Figure 12, the population distribution and the results obtained (red points) are shown at iteration number 500, which satisfies the design constraints. 
Figures 13 and 14 show the evolution of the fitness function and the different evaluated utilization ratios during the multi-objective optimization process.

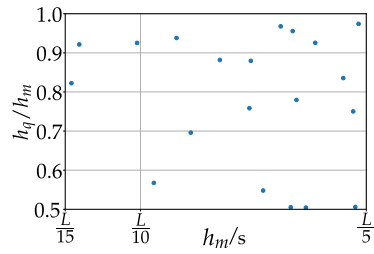

(a)

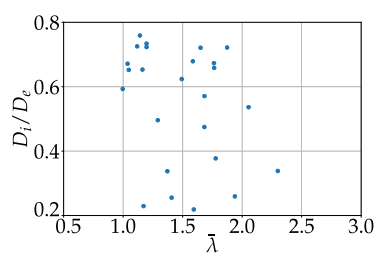

(b)

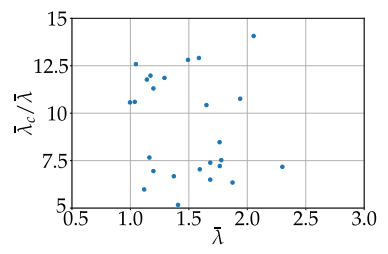

(c)

Figure 11: Initial population

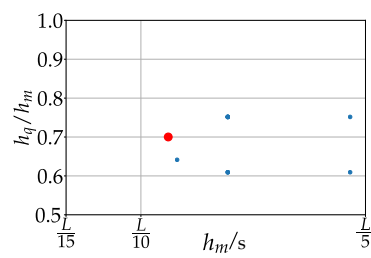

(a)

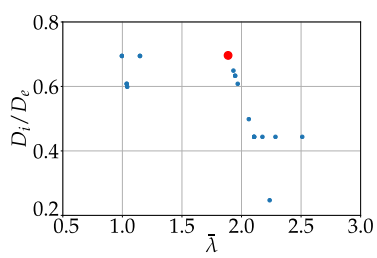

(b)

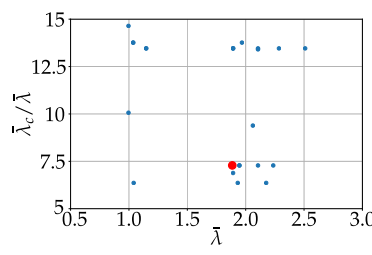

(c)

Figure 12: Distribution of the population and results (red points) at final iteration

Table 4 shows the form-finding variables obtained at different stages of the multi-objective optimization process with geometries shown in Figure 15. Tables 5 and 6 show the utilization ratios of the rod and cables and the maximum span245 deflection ratios for the structural solution reached in the optimization process.

\begin{tabular}{cccccccc}
\hline & Iter & Fitness & $h_{m} / s$ & $h_{q} / h_{m}$ & $\bar{\lambda}$ & $D_{i} / D_{e}$ & $\bar{\lambda}_{c}$ \\
\hline a) & 1 & 0.0549 & 0.393 & 0.925 & 1.767 & 0.673 & 8.47 \\
b) & 5 & 0.0268 & 0.595 & 0.697 & 2.404 & 0.575 & 8.47 \\
c) & 150 & 0.0149 & 0.448 & 0.697 & 1.887 & 0.701 & 7.28 \\
d) & 500 & 0.0148 & 0.447 & 0.697 & 1.891 & 0.695 & 7.26 \\
\hline
\end{tabular}

Table 4: Form-finding variables for different configurations 


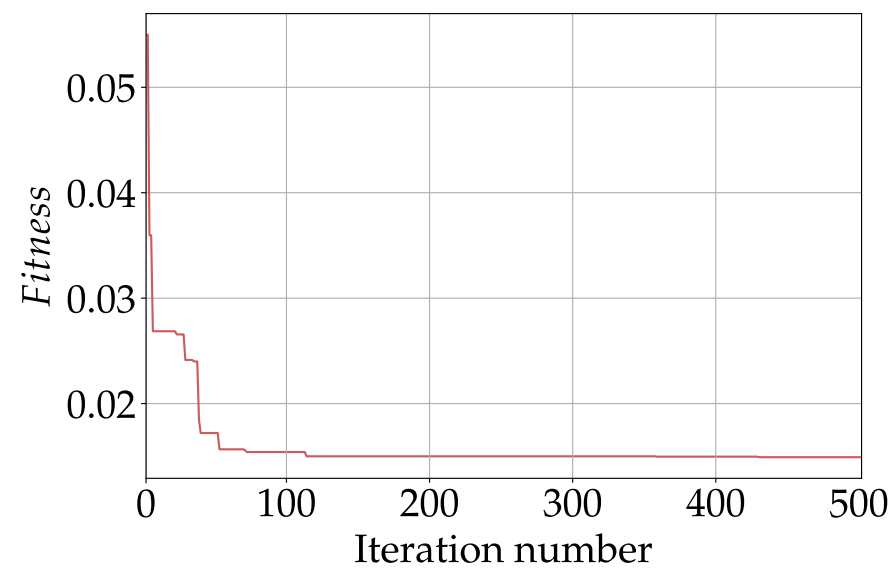

Figure 13: Evolution of the fitness function
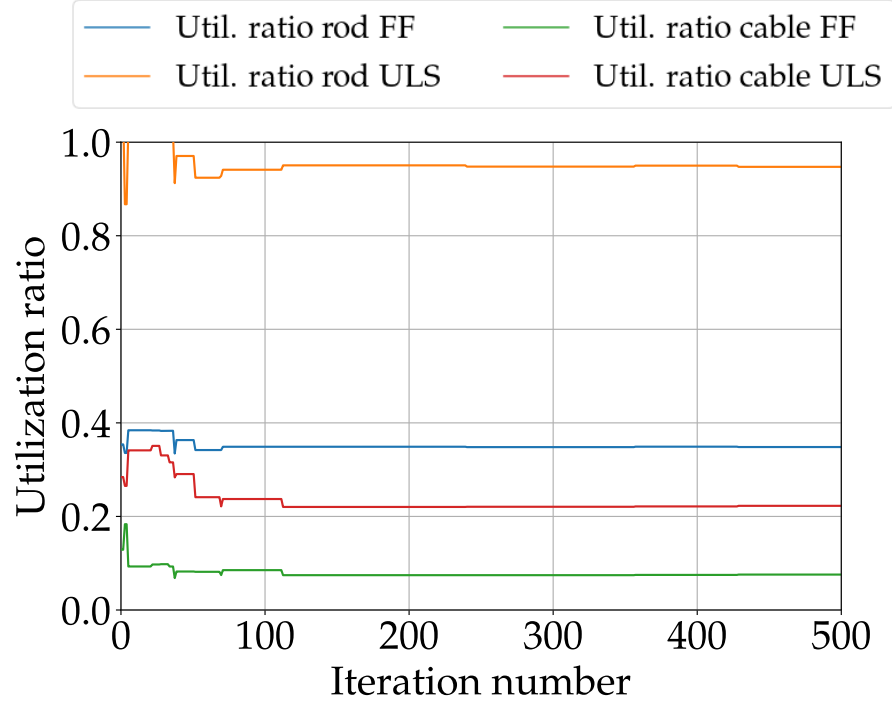

Figure 14: Utilization ratios of the rod and cables after activation (FF) and for the ultimate limit state (ULS) 


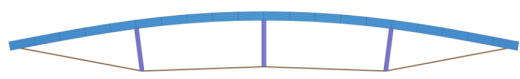

(a) Iteration number 1

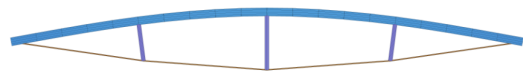

(c) Iteration number 150

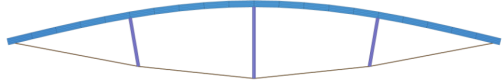

(b) Iteration number 5

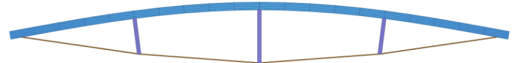

(d) Iteration number 500

Figure 15: Configuration for bending-active tied arches at different iterations

\begin{tabular}{cc|cc}
\hline \multicolumn{2}{c|}{ After activation } & \multicolumn{2}{|c}{ ULS } \\
\hline Rod & Cable & Rod & Cable \\
0.3529 & 0.0771 & 0.9551 & 0.2233 \\
\hline
\end{tabular}

Table 5: Utilization ratios of the rod and cables for the solution reached

As can be seen from the results shown in Tables 4,5 and 6 the genetic algorithm evolves towards efficient solutions characterized by active members with moderate values of slenderness, minimal cross-sections for the ultimate limit state of the structure and cable cross-sections slightly oversized, that provide the required global stiffness to the tied arch, at the expense of a low, but sufficient, cable stress after activation (Fig. 14).

\begin{tabular}{cc|cc}
\hline \multicolumn{4}{c}{ SLS [mm] } \\
\hline$D_{q, \text { sym }}$ & $D_{m, \text { sym }}$ & $D_{q, \text { asym }}$ & $D_{m, \text { asym }}$ \\
2.88 & 3.30 & 0.83 & 2.70 \\
\hline
\end{tabular}

Table 6: Maximum deflections (in $\mathrm{mm}$ ) for the solution reached 
Regarding the cross-sectional shape of the rod, the genetic algorithm tends to converge on solutions with ratios $D_{i} / D_{e} \approx 0.6$. This value maximizes the stiffness and minimizes the cross-sectional area, which favors light structural schemes and supports the assumption made in previous work [14.

The evaluation of the stress in the flexible member after activation is another important aspect in the design of bending-active structures. High stresses can lead to the emergence of long-term strains due to creep in GFRP materials. Consequently, stresses after activation should be within $30 \%-60 \%$ of the maximum allowable stress to avoid this phenomenon [10, 11. In the solution obtained, stresses in the rod after activation are limited to $35 \%$ of the allowable stress, which is a moderate and desirable value. For the evaluation of deflections the limitation posed by the Eurocode for footbridges has been considered. The maximum value for deflections must be lower than $L / 1200$, where $L$ is the span length. In the solution reached, the active member is $4 \mathrm{~m}$ long $L$ and maximum deflections are shown in Table 6. For each value, the limitation $L / 1200$ is fulfilled.

The outcomes of the experiment are expressed in terms of non-dimensional parameters. In previous work [14, authors show that, for a given member 270 cross-section, outcomes can be generalized for flexible members of any length and stiffness, as long as: the shape of the bending-active arch is equivalent. This means that the deviators are equally spaced and perpendicular to the rod; deviator at midspan are $44 \%$ of the developed length of the rod; the length of the other two is $70 \%$ of the central deviator (Table 4 configuration $\mathrm{C}$ ) and the relation between external loads remains constant. For example, for the design of a footbridge consisting of a $12 \mathrm{~m}$ long continuous active member with a riseto-span ratio $f / a$ of $6 \%$, according to the rod slenderness (1.891) and cable slenderness (13.728) obtained in the study, the tied arch can be built using a circular hollow cross-section with an outer diameter of $190 \mathrm{~mm}$ and a thickness 280 of $30 \mathrm{~mm}$ and a steel cable with a diameter of $26 \mathrm{~mm}$. 


\section{Structural analysis and verification}

From a structural point of view, it is crucial to understand why the obtained solution is optimal. This section aims to explain why the algorithm tends to this configuration and how the form-finding parameters influence the structural behavior of the solution. For that purpose, the analysis focuses on the final solution after 500 iterations. In order to better understand the outcomes, the optimal solution is compared with a 'bad' solution (first iteration of the algorithm). In this way, it is possible to appreciate the improvement of the structural solution.

Among the different form-finding parameters, the length of the deviators are the most critical to the behavior of the whole structure. Comparing the structure with a truss girder, the length of the deviator at midspan defines the height of the beam, which directly influences the overall stiffness of the system. As expected, higher values of the deviator length at midspan lead to smaller deflections (Figs. 16 and 17).

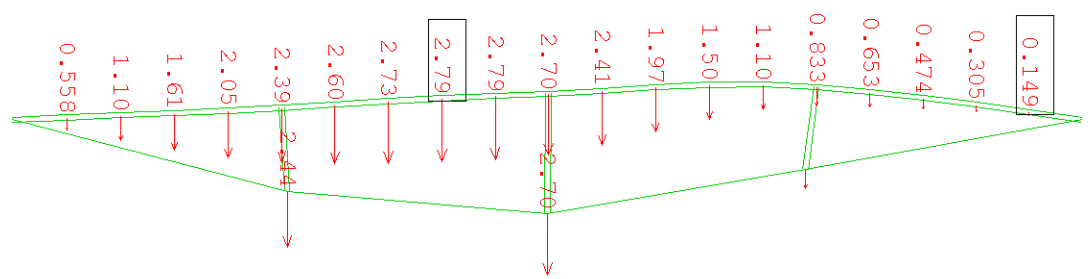

Figure 16: Vertical deflections $(\mathrm{mm})$ due to the non-symmetric loading pattern for the serviceability limit state (optimal solution)

295

Another important aspect is the ratio between deviator lengths $h_{q} / h_{m}$. Figures 18 and 19 show the bending moments in the rod obtained from a FE model at the form-finding stage. As can be seen, for a ratio $h_{q} / h_{m} \approx 0.7$, the maximum value for the bending moment is located at midspan, where higher curvatures are expected, and decreases gradually until zero at the ends. This behavior is the expected structural response in elastica-shaped active arches. 


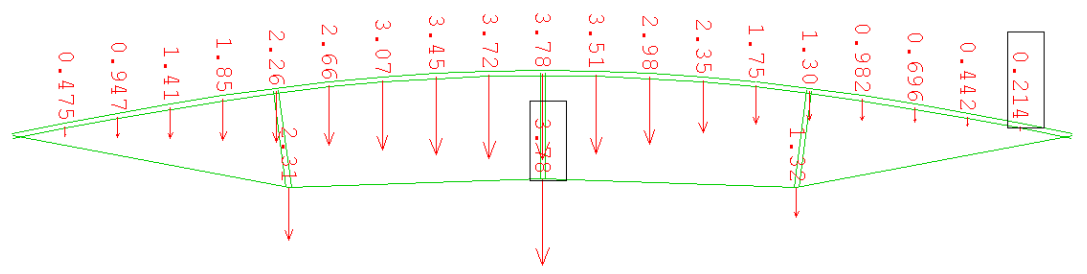

Figure 17: Vertical deflections (mm) due to the non-symmetric loading pattern for the serviceability limit state (bad solution)

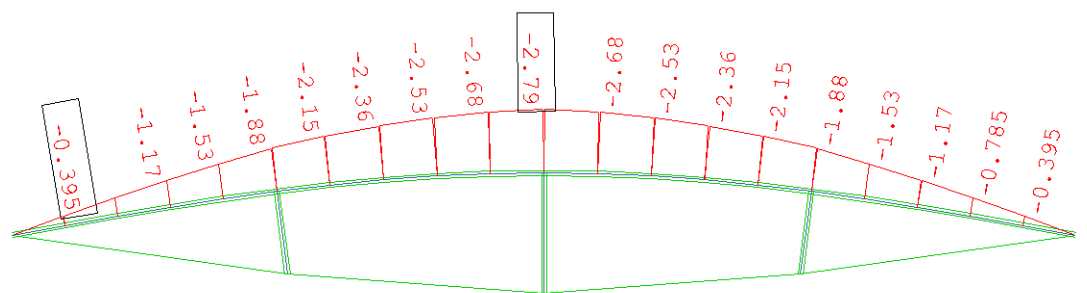

Figure 18: Bending moments $M$ (kN.m) in the rod due to activation process (optimal solution)

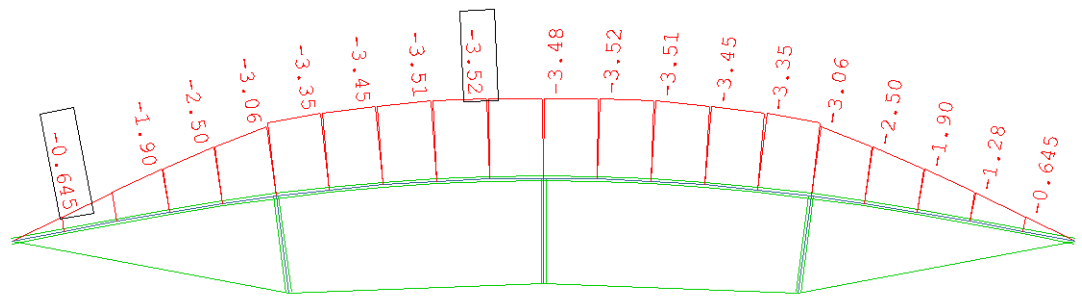

Figure 19: Bending moments $M$ (kN.m) in the rod due to activation process (bad solution) 
As can be observed in Figures 20 and 21 the ratio between deviator lengths $h_{q} / h_{m}$ also influences their behavior. For example, in the configuration corresponding to the 'bad' solution (Fig. 21), the deviator at midspan works under tension, and it could therefore be replaced by a cable. However, this structural scheme is not the most efficient to bear the action of the design loads posed by the Eurocode for footbridges. Therefore, it seems desirable to obtain configurations where deviators work under compression.

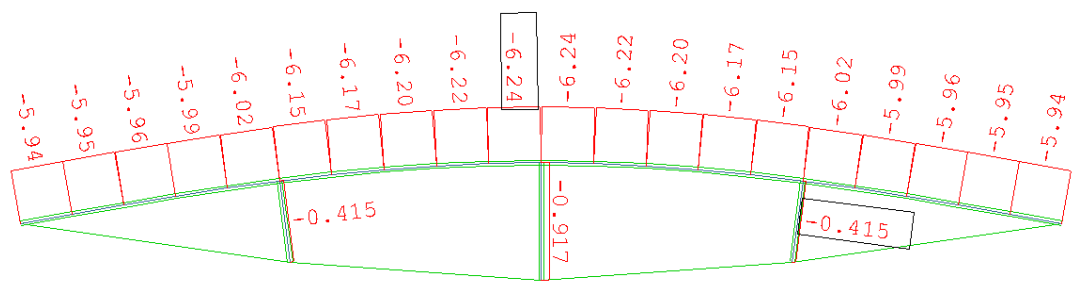

Figure 20: Axial forces $N(\mathrm{kN})$ in the rod and deviators due to activation process (optimal solution)

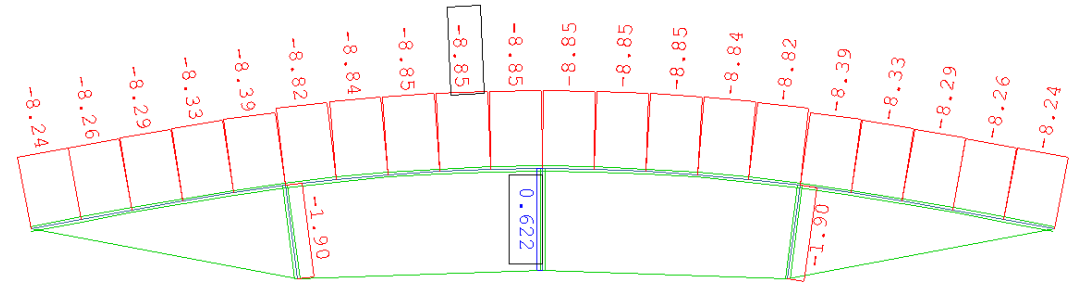

Figure 21: Axial forces $N(\mathrm{kN})$ in the rod and deviators due to activation process (bad solution)

The selection of the cables is also crucial in the behavior of the whole structure. As mentioned in the previous section, solutions are characterized by cable cross-sections slightly oversized. The optimization method evolves towards solutions where cables are designed to avoid cable slackness and provide maximum stiffness to the whole system, since higher values of cable-rod slenderness ratio lead to larger deflections [14]. 

stiffness and minimum cross-sectional area has been reached. As can be seen in Figures 22 and 23 for the ultimate limit state, stresses produced by bending moments play a more prominent part. However, the effect of axial force cannot be neglected due to their contribution to the buckling of the rod segments. In addition, other local phenomena such as crushing or local buckling must be avoided. As expected, the optimization method has driven towards a high value of rod slenderness, reducing as much as possible the external diameter and optimizing the thickness to get an utilization ratio close to 1 (0.9551).

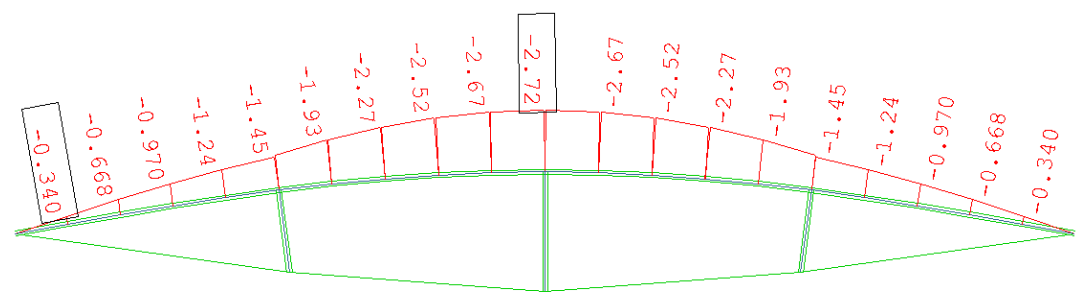

Figure 22: Bending moments $M$ (kN.m) in the rod due to the design loads for the ultimate limit state (optimal solution)

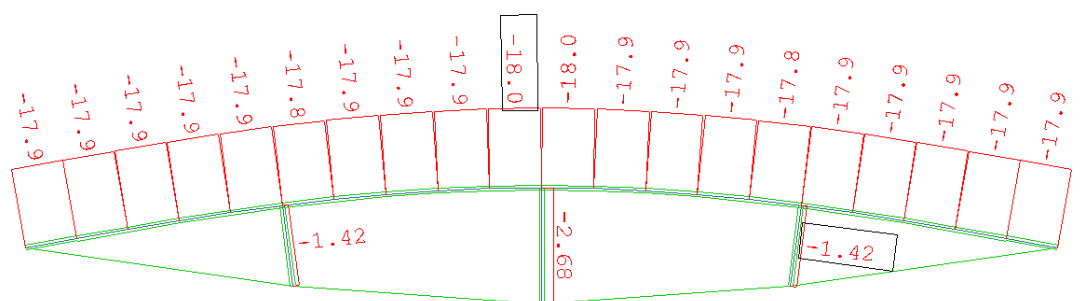

Figure 23: Axial forces $N(\mathrm{kN})$ in the rod due to the design loads for the ultimate limit state (optimal solution) 


\section{Concluding remarks}

325 The design of bending-active structures is a challenging problem. The high non-linearity of the activation process, the coupling between member sizing and their high flexibility make it difficult to design this kind of structures efficiently. This paper presents a design tool based on multi-objective optimization for obtaining efficient structural configurations for bending-active tied arches, experimental resistant schemes to design lightweight pedestrian bridges or roofing applications. The structural optimization has been carried out by a genetic algorithm, in which each plausible structural configuration has been evaluated in terms of stresses in the active member and cables, maximum deflections for serviceability limit state and lightness of the structure, according to the limitations posed by the Eurocode for footbridges. A sensitivity analysis was carried out to improve the performance of the genetic algorithm. It is worth highlighting that it leads to genetic algoritms with small population sizes, which is also advantageous to reduce the computational cost of the simulations. Results are given in terms of non-dimensional parameters to make them applicable to the design of bending-active structures of any size and stiffness. Results show that solutions are mainly dominated by the magnitude of the design loads and limitations for deflections for the serviceability limit state. Less restrictive limitations or a different structural application such as roofing module would allow for wider design alternatives. Section 4 shows an example based on the scalability of the 345 solution. Finally, in section 5 the optimal solution reached by the algorithm has been analyzed and verified from a structural point of view.

\section{Replication of results}

This section shows the core of the PYTHON code developed by the authors for the genetic algorithm presented in this paper. Prior to run the code, each import text data from external tools to build the numerical models. The inputs for the genetic algorithm are given as: 
- $l$ is a number and represents the length of the rod.

- $s$ represents the length of the rod segments between deviators. In this

370 paper this parameter is defined as $l / 4$.

- sol_per_pop is a number and indicates the size of the population.

- $p o p$ must be introduced as a list. It contains the form-finding variables for each individual of the population.

- fitness is a list. It contains the fitness values of the individuals.

Each part of the code is identified by labels to facilitate the reference with the corresponding section in the paper. The original code reads as:

import numpy as $n p$

def roulette_selection(l, s, sol_per_pop, pop, fitness):

\#Fitness proportioning

365

$$
\begin{aligned}
& \text { offspring }=\text { numpy.empty }((\text { sol_per_pop, pop.shape[1] })) \\
& \text { fitness_inv }=[\text { numpy.sum(fitness) }- \text { fitness }[i] \text { for i in range(len(fitness }))] \\
& \text { fitness_inv_sum }=\text { numpy.sum(fitness_inv) } \\
& \text { fitness_proportion }=\text { fitness_inv/fitness_inv_sum }
\end{aligned}
$$$$
\text { intervals }=[]
$$$$
\text { summ }=0
$$

for $\mathrm{i}$ in range(sol_per_pop):

$\mathrm{k}$ = fitness_proportion[i]+summ

intervals.append $(\mathrm{k})$

$$
\text { summ }=\mathrm{k}
$$

\section{\#Selecting parents}

for i in range(sol_per_pop):

roulette $=$ numpy $\cdot$ random $\cdot$ uniform $($ low $=0$, high $=1$, size $=1)$

$$
\text { ParentA_index }=0
$$


for $\mathrm{j}$ in range(len(intervals)):

if roulette $<=$ intervals[ParentA_index]:

break

else:

ParentA_index $=$ ParentA_index +1

roulette $=$ numpy $\cdot$ random $\cdot$ uniform $($ low $=0$, high $=1$, size $=1)$

ParentB_index $=0$

for $\mathrm{j}$ in range(len(intervals)):

390

if roulette $<=$ intervals[ParentB_index]:

break

else:

395

400

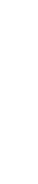

405

410
ParentB_index $=$ ParentB_index +1

while ParentB_index $==$ ParentA_index:

roulette $=$ numpy.random.uniform $($ low $=0$, high $=1$, size $=1)$

ParentB_index $=0$

for $\mathrm{j}$ in range(len(intervals)):

if roulette $<=$ intervals[ParentB_index]:

break

else:

ParentB_index $=$ ParentB_index +1

\#Crossover

CO_num $=$ numpy.uint8(numpy.random.uniform $($ low $=1$, high $=\mathbf{l e n}($ pop $[0]), \operatorname{size}=1)$ )

crossover_point $=\mathrm{CO}_{-}$num $[0]$

offspring $[i, 0:$ crossover_point $]=$ pop $[$ int $($ ParentA_index $), 0$ :

crossover_point] 
offspring[i, crossover_point:] = pop[int(ParentB_index),

crossover_point:]

\#Mutation

415

420

425

430

435

440

for $\mathrm{i}$ in range(sol_per_pop):

$\# 40 \%$ - > high $1-11$

dice $=$ numpy.uint 8 (numpy.random.uniform $($ low $=1$, high $=11)$, size

$=1)$

if dice $<=4$ :

$$
\operatorname{print}(" m u t ")
$$

$m u \_n u m=$ numpy.uint 8 (numpy.random.uniform $($ low $=0$, high $=$

$\operatorname{len}(\operatorname{pop}[0]), \operatorname{size}=1))$

mutation_point $=$ mu_num[0]

if mutation_point $==0$ :

offspring $[i$, mutation_point $]=$ numpy.random.uniform $(1 / 15,1$

$$
/ 5,1)
$$

elif mutation_point $==1$ :

offspring[i, mutation_point $]=$ numpy.random.uniform $(0.3,1$,

1)

elif mutation_point $==2$ :

offspring $[\mathrm{i}$, mutation_point $]=$ numpy.random.uniform $(\mathrm{s} / 20, \mathrm{~s}$

$/ 8,1)$

elif mutation_point $==3$ :

offspring $[\mathrm{i}$, mutation_point $]=$ numpy.random.uniform $(0.2$,

$0.8,1)$

elif mutation_point $==4$ :

offspring[i, mutation_point $]=$ numpy.random.uniform $(5,15$,

1)

\#Elitism 


$$
\begin{aligned}
& \text { index_elitism }=\text { numpy.where(fitness }==\text { numpy.min(fitness) }) \\
& \text { index_elitism }=\text { index_elitism }[0][0] \\
& \text { offspring[sol_per_pop-1] }=\text { pop[index_elitism] } \\
& \text { return offspring }
\end{aligned}
$$

445 Once the genetic algorithm generates the new structural configurations, the process starts again performing a non-linear analysis for each individual using SOFiSTiK.

\section{Acknowledgements}

The authors gratefully acknowledge the financial support from the Span450 ish Ministry of Economy and Competitiveness through grant BIA2015-69330-P (MINECO), the European Union programme through grant ERASMUS Traineeships $2017-\mathrm{E}+$ and the support from CALTER Ingeniería and SOFiSTiK AG for providing a software license.

\section{References}

\section{References}

[1] E. Happold, W. I. Liddell, Timber lattice roof for the Mannheim Bundesgartensschau, The Structural Engineer 53 (3) (1975) 99-135.

[2] O. Baverel, J. F. Caron, M. Beaugelin, J. Bonthoux, S. Martin, Concept of a beam prestressed by bending: Application to a footbridge in composite materials, Journal of the International Association for Shell and Spatial Structures 51 (3) (2010) 99-106.

[3] C. Douthe, O. Baverel, J. F. Caron, Gridshell in composite materials: Towards wide span shelters, Journal of the International Association for Shell and Spatial Structures 48 (3) (2007) 175-180. 
[4] C. Douthe, J. F. Caron, O. Baverel, Gridshell structures in glass fibre reinforced polymers, Construction and Building Materials 24 (9) (2010) $1580-1589$.

[5] S. Pone, S. Colabella, B. Parenti, D. Lancia, A. Fiore, B. D'Amico, F. Portioli, R. Landolfo, M. D'Aniello, C. Ceraldi, Construction and form-finding of a post-formed timber grid-shell, in: Structures and Architecture: Concepts, Applications and Challenges - Proceedings of the 2nd International Conference on Structures and Architecture (ICSA 2013), CRC Press, 2013, pp. 245-252, guimaraes, Portugal, 24-26 July 2013.

[6] P. Nicholas, E. Lafuente-Hernández, C. Gengnagel, The Faraday Pavilion: activating bending in the design and analysis of an elastic gridshell, in: Proceedings of the Symposium on Simulation for Architecture \& Urban Design (SimAUD 2013), Society for Modeling \& Simulation International, 2013, pp. 154-161, san Diego (CA), USA, 7-10 April, 2013.

[7] J. Lienhard, S. Schleicher, J. Knippers, Bending-active structures - research pavilion icd/itke, in: D. Nethercot, S. Pellegrino (Eds.), Proceedings of the IABSE-IASS Symposium - Taller, Longer, Lighter, 2011.

[8] J. Harding, W. Pearson, H. Lewis, S. Melville, The Ongreening Pavilion, in: Advances in Architectural Geometry 2014, Springer International Publishing, 2014, pp. 295-308.

485 [9] J.-F. Caron, S. Julich, O. Baverel, Selfstressed bowstring footbridge in frp, Composite Structures 89 (3) (2009) 489 - 496. doi:https://doi.org/10. $1016 / j$.compstruct.2008.11.009.

[10] J. Lienhard, Bending-active structures: Form-finding strategies using elastic deformation in static and kinetic systems and the structural potentials therein, no. 36 in itke Forschungsberichte, Universität Stuttgart - Institut für Tragkonstruktionen und Konstruktives Entwerfen, 2014. 
[11] C. Douthe, Etude de structures élancées précontraintes en matériaux composites: application à la conception des gridshells, Ph.D. thesis, thèse de doctorat dirigée par Caron, Jean-François Matériaux et structures Marnela-vallée, ENPC 2007 (2007).

URL http://www .theses.fr/2007ENPC0728

[12] C. Lázaro, S. Monleón, J. Bessini, Tangent stiffness in point-loaded elastica arches, in: Proceedings of the IASS Annual Symposium 2017, Hamburg, 2017.

500

[13] C. Lázaro, J. Bessini, S. Monleón, Shape and performance of bending-active tied arches, in: Proceedings of the IASS Annual Symposium 2018, Boston, 2018.

[14] J. Bessini, C. Lázaro, J. Casanova, S. Monleón, Efficiency-based design of bending-active tied arches, Engineering Structures 200, 109681 (2019).

${ }_{505}^{50}$ [15] Y. Sakai, M. Ohsaki, Discrete elastica for shape design of grid-

a shells Engineering Structures 169 (2018) 55 - 67. doi:https: //doi.org/10.1016/j.engstruct.2018.05.002.

a URL http://www.sciencedirect.com/science/article/pii/ S0141029617338695

${ }_{510}$ [16] K. Noda, Y. Kanebako, Structural design of pre-bent dimensional lumber suspenarches, in: Proceedings of the IASS Annual Symposium 2018, Boston, 2018.

[17] J. Bessini, R. Piñol, C. Lázaro, S. Monleón, Design of an experimental lightweight footbridge based on the active bending principle, in: Proceed$515 \quad$ ings of the IASS Annual Symposium 2018, Boston, 2018.

[18] BS EN 1993-1-1 Eurocode 3: Design of steel structures - Part 1-1: General rules and rules for buildings, British Standards Institution, 2005.

[19] BS EN 1991-2 Eurocode 1: Actions on structures - Part 2: Traffic loads on bridges, British Standards Institution, 2003. 
[20] J. McCall, Genetic algorithms for modelling and optimisation, Journal of Computational and Applied Mathematics 184 (2005) 205-222.

[21] O. Hasançeby, F. Erbatur, Evaluation of crossover techniques in genetic algorithm based optimum structural design, Computer \& Structures 78 (2000) 435-448.

525 [22] A. Konak, D. Coit, A. Smith, Multi-objective optimization using genetic algorithms: A tutorial, Reliability Engineering \& System Safety 91 (2006) $992-1007$.

[23] I. Sobol, Uniformly distributed sequences with and additional uniform property, USSR Computational Mathematics and Mathematical Physics 530 16(5) (1976) 236-242. 